\title{
QUAND IL EST FORT EN THÈME : \\ ENTRE DOUBLE MARQUAGE ET ANAPHORE ZÉRO
}

\author{
Guy ACHARD-BAYLE \\ Université de Lorraine \& CREM Praxitexte
}

Lui demeurait à l'entresol. Elle, demeurait au cinquième étage...

(Alphonse Allais, Idylle Moderne)

« [U]n thème est le développement d'une variété. » (SÉMIR BADIR, $2014:$ 1)

\begin{abstract}
En): This paper deals with a "strange case of topicalization" in the poem Idylle Moderne by Alphonse Allais. On the one hand we can find, at the very beginning of the text, an accentuated form of the "pronoun", that is to say a nominal substitute; on the other hand, it seems that the comma of the first line has disappeared, while it is maintained in the latter. Between a "false" double-marking and a zero anaphora, we try to describe the syntactical and the informational organization of the first sentences in terms of a hybrid form of operation, where (and when) a topic plays a focal part too..
\end{abstract}

Keywords (En): Topic-focus ; topicalization-focalization ; double-marking ; zero anaphora ; functional syntax perspective.

Mots-clés $(\mathbf{F r})$ : thème-rhème ; thématisation-rhématisation ; double marquage ; anaphore zéro ; syntaxe fonctionnelle.

\section{Introduction - en forme de court avant-propos - sur deux « points d'ordre »}

Mon premier point d'ordre concerne le titre: pour le colloque, entre la proposition de communication et la présentation orale, j'ai changé de titre : de Quand «Lui » est fort en thème je suis passé à Quand «Il » est fort en thème; je développerai ce point dans ma première partie, car ce changement de titre éclaire largement la problématique et la démarche que j'ai choisies pour traiter ce que je considère - je voudrais le dire d'emblée - comme un cas à part sinon «a-normal » de thématisation :

\section{IDYLLE MODERNE}

Lui demeurait à l'entresol.

Elle, demeurait au cinquième étage.

Lui, était un garçon dans les vingt-cinq ans, pas vilain garçon, mais bête comme tout.

Elle, était une jeune fille dans les vingt-deux ans, plutôt jolie, mais un peu drôle... ${ }^{1}$

Mon second point d'ordre concerne la délimitation du champ : je voudrais en fait dans cet avant-propos mettre un peu d'ordre, c'est-à-dire commencer par «déblayer» le terrain ; je laisserai en effet de côté, c'est-à-dire pour plus tard, l'approche diachronique de la question du détachement thématique : je suis en cela Combettes (1998) qui, lui-même, la traite «in extremis", dans son dernier chapitre; cela ne veut pas dire que cette approche soit secondaire: bien au

\footnotetext{
${ }^{1}$ Au vers 1 , suivant la version écrite, on trouve la virgule ou non ; voir infra l'édition citée du Livre de Poche.

Echo des études romanes XI/1, 2015

ISSN: 1804-8358 (Online)
} 
contraire, du fait qu'elle nous renseigne sur un état de langue ${ }^{2}$ où le phénomène, qui dans les vers cités résulte d'une thématisation, et que nous allons étudier sous la forme de ce que j'appellerai anaphore zéro, se révèle assez massivement attesté au $\mathrm{XVI}^{\mathrm{e}}$ siècle encore. ${ }^{3}$

Je remets également à plus tard une étude, ou une vérification, quantitative (basée sur corpus), rendue nécessaire par la rareté de l'occurrence qui nous intéresse ici ; de même, je laisserai de côté une étude contrastive avec une forme de thématisation proche : «Quant à lui, ... »; cette forme, outre qu'elle est peu probable à l'initiale d'un texte, ne tolère, à l'inverse de celle que nous étudions pour l'heure, ni absence de virgule, ni absence de redoublement pronominal.

Je me centrerai donc dans cet article sur ce qui me semble être, vis-à-vis l'état de langue ou de l'usage concernés, un cas à part, ou «a-normal » comme je le disais d'entrée ; mais il me faut désormais en dire un peu plus : il me semble en effet qu'on a ici affaire à une forme de double marquage $e^{4}$ rare sinon inédit du fait que ce double marquage s'opère d'une part avec «défaut», d'autre part «par défaut », c'est-à-dire avec, d'un côté, une virgule «variable », de l'autre, une ellipse du pronom, autrement dit une anaphore zéro. ${ }^{5}$

\section{Sur un changement de titre et de thème : de $\mathbf{L u i}$ à $I l$}

Ce changement de titre me permet de faire la démonstration directe, ou pratique, de mon propos. ${ }^{6}$ Ainsi, la première chose que je peux dire, c'est que si j'ai finalement choisi comme titre Quand «Il» est fort en thème au lieu de Quand «Lui » est fort en thème, c'est que ce changement me permettait de mieux jouer sur la double valeur référentielle et donc informative de $I l / i l$ : dans un cas, c'est-àdire dans le cas où «il » est en usage, il l'est comme substitut pronominal d'un référent ; comme par ex. dans : «Pierre, il est fort en thème. »; dans un autre cas, celui où il est en mention, et c'est le nôtre, « Il » (avec majuscule) est utilisé comme marque référentielle autonymique. Or, à chaque cas correspond un schéma prosodique : dans le second cas «Il» est rythmiquement isolé, ou délié ${ }^{7}$, et

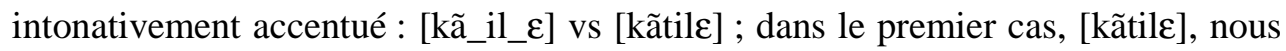
avons, autour de «il », deux liaisons consonne-voyelle : [kãtil] et [ile].

La seconde chose que je voudrais dire ou rappeler, après ce test sur « Il » et « il », c'est que le substitut pronominal « il »étant «faible » ou «atone », il lui

\footnotetext{
${ }^{2}$ Et un niveau ou un registre de langue : littéraire - puisqu'on ne dispose que de lui, c'est-à-dire de ces sources écrites...

${ }^{3}$ Voir COMBETTES (op. cit.) ou encore DuFRESNE (1995).

${ }^{4}$ Sur marquage et double marquage, voir BlAnCHE-BENVENISTE (1997), BlanCHE-BENVENISTE et al. (1990), Blasco-Dulbecco (1999), Klingler (2011) et Veronique (2013).

${ }^{5}$ Voir notamment CoRNish (2001:5) : «Pour KLEIBER [1994, sans mention de page], l'anaphore pronominale (et, nous pouvons ajouter, l'anaphore zéro) répond à trois conditions essentielles [dont je retiens les deux premières] : (1) elle suppose partagée la représentation d'une situation saillante dans laquelle (2) un protagoniste central ressort de l'arrière-plan contextuel... »

${ }^{6}$ Sans mauvais jeu de mot, métalinguistique... Comme quoi, que «mon propos soit de parler du thème » montre que même à un niveau métalinguistique, la syntaxe fonctionnelle... fonctionne !

${ }^{7}$ Voir infra nos références à COMBETTES (1998).
} 
faut, pour prendre de la voix, de l'accent et du rythme, et gagner ainsi en tonicité, autrement dit pour devenir «fort en thème », se changer en «Lui ». La question que pose néanmoins cette métamorphose, suivant la norme actuelle et même de quelques siècles passés ${ }^{8}$, c'est qu'en fait de métamorphose, s'opère généralement, i.e. dans l'usage courant, depuis quelques siècles du moins en français, un dédoublement; d'où l'étrangeté de cette métamorphose totalement accomplie si on peut dire, puisqu'on y voit l'entité dédoublée, ou parallèle, disparue :

\section{Lui (,) (il) demeurait...}

Je dis bien «entité parallèle » et non «entité d'origine», pour «il», car je considère, avec COMBETTES (1998), mais aussi BLANCHE-BENVENISTE (1997), que la thématisation par double marquage ${ }^{9}$ n'est pas la «transformation » d'une proposition supposée « de base »:

\section{Il demeurait... $\Rightarrow$ Lui, il demeurait...}

Tant il est vrai, notamment en français oral ${ }^{10}$, que cette structure (« syntaxique fonctionnelle ») de l'énoncé au pronom «dédoublé » serait plutôt la «norme », suivant une fois encore l'usage ordinaire.

Pour autant, c'est-à-dire quand bien même on refuse la notion ou l'opération de «transformation », dans le sens dérivationnel ${ }^{11}$ qu'a pu lui donner la grammaire générative, on ne peut pas ne pas mettre en correspondance ou en parallèle, suivant entre autres COMBETTES (1998:10), les deux énoncés et leur structure :

Il demeurait... // Lui, il demeurait...

Donc, nous dirons, encore avec COMBETTES (1998: 10), qu'on a dans ce cas de thématisation, d'une part «une structure liée», de l'autre une structure « segmentée et déliée ».

Et dès lors que, dans notre exemple de départ, on reconnaît en «Lui » d'une part un sujet (grammatical, sémantique ou encore informationnel), d'autre part un sujet en position détachée (toujours selon COMBETTES, 1998), il nous faut interroger ce statut de sujet détaché ou délié, dans la mesure où, répétons-le, «l'absence» de son alter ego, le substitut clitique atone «il », étonne; du moins étonne-t-elle le lecteur ou le locuteur d'aujourd'hui...

\footnotetext{
${ }^{8}$ Norme qui est pour moi ici celle de l'usage, et non celle de la grammaire «normative » qui condamne la redondance supposée familière qui résulte de cette forme de thématisation...

${ }^{9}$ Voir supra note 4.

${ }^{10}$ Voir là encore Blanche-Benveniste (1997) et BlanChE-BENVENISTE et al. (1990).

${ }^{11}$ C'est-à-dire suivant un modèle qui considère qu'il y a une base qui d'une manière ou d'une autre sert de modèle, alors que l'usage, oral en tout cas, tend à montrer l'inverse ; pour une caractérisation princeps de la GGT (grammaire générative transformationnelle ou « dérivationnelle »), voir DuBoIS (1969).
} 
C'est qu'en effet, pour le lecteur d'aujourd'hui, fût-il linguiste, mais coupé de l'histoire de la langue et des normes attachées aux genres ${ }^{12}$, il est difficile de reconnaître précisément la fonction de ce sujet ; c'est-à-dire, sa motivation, et son effet.

Mon propos est donc maintenant d'analyser cette forme marginale ou «périphérique $»^{13}$ en répondant à une série de questions qui devraient nous permettre de cerner le statut informatif du pronom tonique : thématique ou non ; ou bien, plus ou moins thématique ; ou enfin, « hybride » : thématico-rhématique ?

Je pourrai décliner cette interrogation comme suit, dans un ordre non pas aléatoire, mais «vagabond», comme on dirait de mouvements qui procèdent, méthodologiquement parlant, par «thème et variations ${ }^{14}$ :

- Dans quelle mesure ces pronoms toniques non dédoublés, qui plus est non systématiquement détachés, mais utilisés en première mention, peuvent-ils être considérés comme des thèmes ?

- Si oui, comment pourrons-nous considérer le changement thématique d'un énoncé à l'autre, d'un énoncé vers l'autre, si nous étudions la forme avec « dédoublement » de ces énoncés?

- Quoi qu'il en soit, s'il y a thème (ou thèmes), il y a présupposition (MARTIN, 1983) : dans ce cas, à quoi ce ou ces thèmes répond(ent)-il(s) ?

- Autrement dit : que rappelle(nt)-t-il(s), au sens mémoriel du verbe...

- Mais aussi au sens échoïque ; autrement dit : à quoi fait/font-il(s) écho ?

- Le recours aux notions de frames ou de schémas de la pragmasémantique ou de la linguistique cognitive suffira-t-il à justifier le choix de ce type d'anaphore (s'il s'agit d'une) au statut mémoriel ou d'accessibilité si particulier, c'est-à-dire si étrange ou inhabituel en première mention ?

- Pour finir, et nous recentrer sur la question de la «norme», nous nous demanderons si au regard de l'usage dominant, on peut parler de double marquage et/ou d'anaphore zéro.

Nous aurions là, sinon une issue, du moins une ouverture : la combinaison des deux opérations - l'hybridité qui en résulterait pour la forme ou l'usage - ne serait pas le moindre paradoxe, mais sans doute une solution adéquate, propre à l'esprit «fantaisiste » de l'écrivain...

Je ne prendrai pas en compte toutes les questions précédentes dans cet article : car si je viens de parler d'ouverture, c'est qu'avec la dernière question et la solution envisagée, s'ouvre une perspective : la question suivante, ou future, que pose en effet ce texte, i.e. ce type ou genre de texte, que j'ai identifié pour ma part

\footnotetext{
${ }^{12}$ D'où la nécessité d'une approche aussi diachronique chez ComBETTES (1998) - à laquelle je me plierai moi-même ; mais, comme je l'ai dit en introduction, une autre fois...

${ }^{13}$ Je reprends ici le thème et les termes d'un des colloques de la série Théories et concepts du Cercle Linguistique de Prague : voir RADIMSKÝ (ed. 2010).

14 «Développement d'une variété [voir notre exergue supra] peut signifier trois choses : que la variété se développe; que la variété développe quelque chose (par exemple, un invariant) ; que quelque chose ou quelqu'un (un texte, un sujet) développe la variété. » (BADIR, art. cit. $2014: 1$ ).
} 
d'emblée comme poétique ${ }^{15}$, est de savoir si on a affaire à un cas de «déviance textuelle », comme dit ADAM (1997: 133) ${ }^{16}$.

Ainsi je projette une seconde approche, qui mettra en avant des questions qui relèvent de la dominante ${ }^{17}$; autrement dit, qui relèvent de la diversité et de la répartition, ou de la hiérarchie des fonctions dans le texte littéraire et plus exactement poétique ; ou encore, pour paraphraser JAKOBSON (1977), qui relèvent de la grammaire de la poésie...

En dehors donc des compléments (diachronique, quantitatif et contrastif) que j'annonçais dans mon introduction, on peut dire maintenant que la première approche fonctionnelle ou textuelle que je propose ici, de la micro- à la macrosyntaxe, à la manière de Daneš, Firbas, mais aussi Combettes ou Adam ${ }^{18}$, ou encore Blanche-Benveniste ou Berrendonner, devra être suivie d'une approche poétique à la Jakobson ${ }^{19}$, mais aussi Chambers, ou encore Adam et Monte.

\title{
2. Analyse formelle, fonctionnelle et textuelle : de la micro- à la macrosyntaxe
}

Je commencerai par poser la question: «Lui» est-il dans ce vers une «construction détachée »? Dans la version écrite du texte citée sur Internet (voir Webographie) :

\begin{abstract}
IDYLLE MODERNE
Lui demeurait à l'entresol.

Elle, demeurait au cinquième étage.

Lui, était un garçon dans les vingt-cinq ans, pas vilain garçon, mais bête comme tout.

Elle, était une jeune fille dans les vingt-deux ans, plutôt jolie, mais un peu drôle...
\end{abstract}

«Lui » au vers 1 n'est pas graphiquement détaché; il l'est par contre au troisième vers.

On trouve dans d'autres éditions (notamment dans Allais... grement, Le Livre de Poche $\left.n^{\circ} 1392,1965\right)$, la virgule après le premier «Lui », comme après tous les substituts qui suivent dans les trois autres vers, le second «Lui » et les deux «lle »...

Il semble donc régner quelque «désordre » à l'écrit; comme d'ailleurs dans les deux versions orales trouvées sur Internet, lors de la préparation de cette étude (mai 2014) : ces lectures à voix haute accentuaient plus ou moins le détachement de «Lui » par la montée mélodique et la pause avant le syntagme verbal suivant;

\footnotetext{
${ }^{15}$ Ceci devra ensuite être justifié ; en attendant, je ferai remarquer que j'ai trouvé, sur le site où j'ai pu télécharger et écouter les versions écrites et orales du texte d'Allais (URL hélas désactivée depuis), sous la rubrique «linguistique des genres populaires", la mention des types ou genres suivants : «poèmes », «nouvelles », « contes » ou encore «poèmes humoristiques ».

${ }^{16} \mathrm{Il}$ le dit à propos d'un poème de Desnos, Maudit soit l'homme (voir Webographie), dont Adam rappelle avant tout « la forme orale populaire ».

${ }^{17}$ En référence à JAKOBSON (1977).

${ }^{18}$ Sur la diversité et la continuité de ces écoles, et l'héritage en France du Cercle Linguistique de Prague, voir ACHARD-BAYLE (éd. 2010) et ACHARD-BAYLE \& CHABROLLE-CERRETINI (éds. 2013).

${ }_{19}$ Ceci constituera en fait un bond rétroactif dans l'histoire des idées ou des phases du Cercle Linguistique de Prague : voir PEŠEK (2010).
} 
je me suis alors demandé si ces lectures orales correspondaient aux deux graphies du vers $1 \ldots$

À défaut de pouvoir répondre à une telle question, nous nous pencherons sur les différents schémas structurels et prosodiques que ces versions impliquent: nous pouvons tout d'abord supposer, du moins dans la version écrite où le premier «Lui » n'est pas suivi d'une virgule, quand le second l'est, que le «désordre » dont je parlais est une «variation »; mais cette dernière ne serait alors que l'effet d'une hésitation que nous pourrions considérer, elle, comme la marque (à peu près certaine) d'une instabilité suscitée par le statut complexe du pronom «sujet», au plan sémantique (discursif ou informationnel) comme au plan logique (ou syntaxique).

Les variations orales quant à elles se justifieraient par le fait qu'il est difficile de lier le pronom tonique au prédicat, particulièrement quand la virgule est absente du vers 1 : la difficulté est alors de respecter la graphie, c'est-à-dire de «faire comme si » le prédicat régissait en fait un clitique, tout en harmonisant cette lecture «liée » de la première structure avec les trois vers suivants où les « forts en thème » figurent détachés, à l'initiale.

Il faut alors ajouter à la liste des questions qui concluaient ma première partie, la suivante : au plan formel, mais aussi communicatif, comment peut-on analyser la structure de chacun des vers, en suivant notamment les trois niveaux d'analyse (syntaxique, sémantique, fonctionnelle ou informationnelle) que recommande COMBETTES dans son ouvrage « pragois » (1983) ?

J'ajouterai toutefois un niveau d'analyse, logique, entre le niveau syntacticosémantique d'un côté et le niveau fonctionnel-informationnel de l'autre. À vrai dire, c'est ici toute la question, et toute la difficulté. A priori, «Lui » est sujet syntaxique et sémantique ; mais qu'est-il du point de vue fonctionnel ? C'est que le statut fonctionnel mais aussi d'une certaine manière logique de «Lui », c'est-àdire son statut prédicatif (extraprédicatif ou non : cela reste à déterminer, et nous $\mathrm{y}$ revenons plus bas et plusieurs fois, en posant la question en termes de double prédication) pourrait remettre en cause l'idée même de sujet syntaxique et donc sémantique.

Pour tenter de résoudre cette difficulté, formelle et sémantique, prédicative ou propositionnelle et textuelle, je m'appuierai de nouveau sur l'ouvrage de COMBETTES (1998) qui traite des constructions détachées, mais également sur BLANCHE-BENVENISTE (1997) et BLANCHE-BENVENISTE et al. (1990), qui se réfèrent essentiellement à la functional linguistics de Halliday.

Repartons donc de COMBETTES (1998): au tout début de son ouvrage (op. cit.: 10), l'auteur constate qu'il y a une grande diversité des structures «frontales »; autrement dit de «constituants en position frontale séparés par une virgule » du reste de l'énoncé. Combettes cite par exemple :

Moi, je m'ennuie.

Avant de poursuivre l'analyse de Combettes sur le statut et la définition du détachement, faisons une première remarque, ou posons une nouvelle question, qui fait écho à l'une de celles restées en suspens en fin de première partie : si, dans 
l'exemple ci-dessus, «je » est sujet de la prédication, en quoi «moi » le serait au même titre, voire à sa place ? Nous y reviendrons sans tarder.

C'est à ce point que Combettes considère à son tour (après BLANCHEBENVENISTE, 1997) l'ambiguitté du terme détachement : ce terme laisserait penser qu'on a « au départ » (COMBETTES, op. cit. : 10) « une structure liée, qui se trouve segmentée, brisée » (ibid.) ; ceci, comme on l'a dit, « impliquerait une structure de base plus simple, d'où serait dérivée la structure avec construction détachée » (ibid.). La thèse de la « dislocation » n'est donc tenable ni au plan théorique ni au plan «psycholinguistique» (ibid.). Je reviens ainsi, au plan théorique, à ma dernière question: pourquoi ou en quoi aurait-on deux sujets ? Dès lors, dire qu'on aurait deux types de sujet semble être une piste à explorer.

On peut s'interroger aussi sur le niveau «psycholinguistique »: il concerne à la fois (i) ce que Combettes appelle «l'ouverture de la phrase », autrement dit le support ou l'à propos de la prédication, et donc (ii) le statut ontologique du référent représenté : en effet, à partir du moment où, dans l'exemple de Combettes, « Moi » est ainsi mis en place, au tout premier plan (et non pas au second, ce qui colle assez mal avec l'idée d'une importance moindre du support vs de l'apport de la prédication), il acquiert un statut mixte : référentiel et sui-référentiel; je veux dire par là que le sujet parlant ou représentant se représente aussi lui-même comme sujet logique : ce sur quoi (subjectum) porte la prédication...

Il me semble trouver le même phénomène de dédoublement référentiel dans le ça déictique (démonstratif contextuel) et résomptif (anaphorique cotextuel) de :

Mais non je ne te parle pas de ça!

Si nous revenons à notre propre exemple, et si nous comparons «Lui » et « Moi », on voit immédiatement ce qui fait l'originalité de notre «Lui »; car quel que soit l'état diachronique ou le niveau sociolinguistique de la langue ici en usage, on ne pourrait avoir :

*Moi, m'ennuie...

Par ailleurs, si «Lui » peut être graphiquement détaché, il peut aussi, au niveau prosodique, i.e. oralement, et donc au niveau conceptuel, i.e. en termes cognitifs ou psycholinguistiques, être ou rester lié au prédicat à des degrés divers, comme dans les différentes versions orales que l'on a pu écouter sur Internet: chacun/e peut s'y essayer en modulant la longueur de la pause entre «Lui » et sa suite.

On a donc affaire à une construction frontale certes, mais au statut de détachement variable : plus ou moins liée par la prosodie, et donc, au niveau de la prédication, par les relations logiques, ou l'intention psycholinguistique. Dans le cas où le pronom « frontal » est lié par la prosodie au prédicat (il est alors à la fois moins accentué et moins détaché), je considérerais qu'il fonctionne comme un nom propre, ce qui du point de vue logique et pragmasémantique (voir KRIPKE, 1972 et ARIEL, 1990 et à leur suite KLEIBER, 1981, 1994, 2001), est tout à fait 
envisageable; que l'on prononce ainsi notre premier vers, avec ou sans pause, avec un accent italien, on le comprendra comme :

\section{Louis(,) demeurait...}

Mais, une fois encore, il s'agit ici plutôt d'une interprétation d'acteur ou de lecteur à voix haute, puisque, du moins dans notre texte d'origine, la virgule est « facultative » à l'écrit. Nous revenons ainsi à la «norme de l'usage » ${ }^{20}$, c'est-àdire à «Lui » détaché par la virgule.

Du point de vue de la «norme de l'usage » d'aujourd'hui donc, notre «Lui » présente diverses autres particularités; celles-ci s'expliquent si l'on considère différents statuts de détachement, autrement dit si l'on «fait le tri » entre divers types de constructions «déliées », et le départ entre les «déliées » et les « détachées » (toujours suivant COMBETTES, 1998).

La première particularité de notre «Lui » est de ne pas être pas mobile, contrairement aux constructions qui peuvent se trouver (i.e. être détachées) sur trois sites : initial ou «frontal », médian et final; autrement dit, pour ce qui nous concerne, occuper les positions pré- et postverbales :

\section{Je m'ennuie, moi...}

Il demeurait, lui...

*Demeurait, lui...

Une seconde différence tient au fait que beaucoup de constructions détachées stricto sensu selon COMBETTES (op. cit. : 11) « sont en relation avec le sujet de la phrase » en tant que circonstants; or, dans les deux exemples précédents, ni «Lui » ni « Moi » ne l'est. C'est que la construction détachée, telle que la définit, donc la retient mais aussi la restreint Combettes (et il en exclut effectivement les pronoms toniques détachés), est un constituant «périphérique », qui n'est «pas syntaxiquement dépendant » (ibid.) ${ }^{21}$.

Ceci posé, on a vu effectivement que «Lui », avec ses lectures variables, peut rester lié donc attaché au prédicat. Pour autant, en fonction toujours des lectures, il peut représenter, comme on l'a dit aussi, divers types de sujet... Reste à savoir lequel ou lesquels, pour introduire ou réintroduire la notion d'hybridité : on vise par là une seconde phase de l'analyse formelle, plus macro- que micro-syntaxique.

Auparavant, faisons un premier bilan, suivant le premier critère de détachement, et les tests qui allaient avec, formels ou structurels : «Lui »ne répond pas à la définition «positionnelle» de la construction détachée selon Combettes, en ce qu'il n'est pas mobile. Ceci dit, si «Lui » n'est pas (un circonstant) «périphérique », il n'est pas toujours (suivant le schéma prosodique adopté) « syntaxiquement dépendant » (COMBETTES, op. cité : 11); ce qui conduit

\footnotetext{
${ }^{20}$ « Norme », mais qu'à moitié : puisqu'il y aurait au regard de la «norme » d'aujourd'hui anaphore zéro.

21 «On peut ainsi légitimement opposer construction détachée et construction liée », ajoute COMBETTES (ibid.). Mais « opposer » n'est pas « dériver », comme on l'a dit plus haut.
} 
à se demander s'il est un constituant «non périphérique dépendant». Ceci pose, ou plutôt repose tout d'abord, à partir et au-delà de la conception de Combettes, la question de ce que l'on entend par détachée, dans construction détachée, et audelà par détachement ; ensuite, la question, qui peut servir à définir une autre des caractéristiques de notre «Lui » : est-il extraprédicatif ?

La première chose que l'on peut dire à ce sujet, c'est que «Lui » (mais ce n'est pas le cas de «Moi » ci-dessus) a encore un comportement différent du fonctionnement de la construction détachée suivant la définition de Combettes : il n'est pas «en relation avec le sujet », il est le sujet, étroitement lié au verbe dans au moins une version orale du texte.

Il résulte de cette définition restreinte de la construction détachée, que si celleci est en relation avec le référent sujet, mais n'est pas le sujet, elle est une forme de prédication seconde; elle a notamment une fonction «parenthétique » lorsqu'elle est détachée au centre de la prédication: elle est dans ce cas «une incise qui apporte une nouvelle information » (COMBETTES, op. cit. : 12). De la sorte, on peut dire non seulement que la construction détachée a une relation implicite avec le sujet mais qu'elle introduit une relation «sous-jacente sujet/prédicat» (COMBETTES, ibid.) ; comme dans les deux énoncés suivants :

Fou de joie, il n'a pas pu répondre.

Il était fou de joie. Il n’a pas pu répondre.

Pour Combettes, la construction détachée est donc une prédication seconde, en ce que «cette prédication seconde vient s'ajouter à une prédication première, principale » (ibid.) ; Combettes parle aussi (ibid.) de «prédication qualifiante» pour la construction détachée, qui est donc « de nature non référentielle ».

Nous retrouvons ici les caractéristiques de notre pronom, ou nous revenons à ses particularités, car notre «Lui » est référentiel et, de par sa position non mobile ( frontale »), se trouve dans la prédication principale, régi par le verbe de cette prédication.

La question reste donc de déterminer le ou les rôles «sujet» que joue «Lui »... On peut dire tout d'abord qu'en l'absence de clitique, «Lui » joue incontestablement le rôle de sujet grammatical : il répond de la manière que le ferait « Il » à la question : «Qui... ? ».

On peut en faire ou refaire le test car il en résulte des enseignements importants :

- $\mathrm{Si}$ «Lui demeurait...» se prête d'un point de vue ou dans une démarche métalinguistique à la question «Qui demeurait...? », en situation (dialogique), il répond ou peut répondre à la même question : «-Qui demeurait...? - Lui demeurait...»

- Il prendrait alors un accent de focalisation qui signifierait quelque chose comme : «C'est lui et non pas elle qui demeurait... » 
Or, si dans notre exemple original, «Lui » a sans conteste une fonction topicale $^{22}$, notamment en position franchement détachée ou déliée (i.e. avec virgule), il n'en est pas moins pris et compris dans un rapport parallèle et contrastif avec « Elle »; et cela à deux reprises.

Un autre élément doit alors être pris en compte : «Lui » apparaît en première mention, et comme de plus il ne rappelle rien de ce qui précède, en l'occurrence le titre, cela pose ou semble poser, conventionnellement ${ }^{23}$, un problème d'accessibilité (dans les termes d'Ariel). Mais le problème est vite résolu du point de vue inférentiel.

De ce point de vue, précisément, on peut imaginer aisément quel scénario (frame), le titre installe dans le programme de lecture de l'interprétant. Dans ce cas, «Lui » aurait bien une fonction de rappel qui à l'oral justifierait parfaitement, quelle que soit la pause, un accent particulier, qui lui-même justifierait la forme tonique.

Mais ce rappel est en même temps une installation en discours, une première mention avons-nous dit, et cette opération lui donne une dimension ou une fonction focale, que vient ensuite confirmer le rôle contrastif du double parallélisme « Lui » vs «Elle».

C'est sans doute cette fonction focale qui pourrait nous rapprocher de ce que Combettes appelle prédication seconde : il s'agit avant de parler de tel ou tel référent, d'introduire ces référents en discours. Quand bien même le poète refuse de passer par la convention de l'indéfini ${ }^{24}$.

Je résume : il nous est apparu jusque là que «Lui » employé seul, c'est-à-dire sans dédoublement par le clitique, qu'il soit détaché ou non, joue plus qu'un rôle topical.

D'un côté, «Lui » est incontestablement «le point de départ », donc de ce point de vue un «topique » (COMBETTES, op. cit. : 56-57 et 138); il est de ce fait le support de la prédication suivante, c'est-à-dire le sujet logique du prédicat à suivre, mais cela ne veut pas dire le support ou le sujet d'une prédication seconde au sens de Combettes ci-dessus: une incise, une parenthèse, une information associée au sujet. D'un autre côté, «Lui » est aussi un point de départ inédit de la narration de l'histoire elle-même inédite : avant de parler de «Lui », il faut l'introduire ou le présenter.

On sait par ailleurs combien le pronom clitique atone est peu utile aux niveaux sémantique et informatif dans d'autres langues romanes; on doit donc accorder au «Lui » tonique un rôle informatif certain, ne serait-ce que contrastif (cela marcherait également avec un «Louis » accentué non délié).

Pour avancer, et finir, je poserai une dernière question, qui ne relance pas la piste de la double prédication, mais lui est conséquente : «Lui » est-il au premier ou au second plan? On aborde alors la question de la logique référentielle de ce genre de texte - et dans ce genre de texte. Cette phase ultime de la démonstration

\footnotetext{
${ }^{22}$ Voir entre autres THEISSEN (2001).

${ }^{23}$ Voir en Webographie d'autres «Idylles » d'Allais qui jouent avec ce schéma ou ces conventions de saisie référentielle en première mention.

${ }^{24}$ Là encore, voir les autres « Idylles » en Webographie.
} 
servira de transition à la seconde approche que j'annonçais, au traitement poétique de ce texte et de son incipit.

Nous avons vu que selon COMBETTES (op. cit. : 13-14), la construction détachée en tant que telle ne réfère pas : «elle n'est pas elle-même une expression référentielle » mais «entraîne la présence d'un référent sous-jacent »; plus loin (op. cit. : 35), Combettes parle d'anaphore sous-jacente.

On voit tout ce que le pronom tonique détaché a de différent, de divergent, avec ce type de construction détachée «classique»: il est endophorique, cataphorique dans le cas où il est en relation avec ou rappelé par un clitique. Mais, seul, il est aussi anaphorique ou encore exophorique : on atteint là un autre niveau ou un autre type de référence, celle des mondes possibles ; autrement dit dans les mondes possibles, aux mondes possibles.

On peut donc, pour aller vers la fin de cette démonstration textuelle, et annoncer la prochaine, plus poétique que formelle ou fonctionnelle, imaginer le scénario suivant qui permet, en guise de seconde synthèse, d'investir pleinement le niveau textuel et de le rendre indissociable des niveaux d'analyse syntaxique et sémantique :

«Lui » est au cœur ou au centre (vs périphérie) d'une prédication première, qui est à la fois de re et de dicto, c'est-à-dire qui (de re) introduit une expression qui réfère «effectivement » («Il existe un $\mathrm{X}$ »); mais qui réfère dans un monde inconnu, sinon supposé connu, ou encore dans un monde de fiction, et donc qui se présente aussi (de dicto) comme un acte de parole narratif fictionnel (c'est-à-dire instaurant la fictionnalité) du type : Il était une fois un $X$, ce $X$ ou X. Faisons comme si (ce) X...

Le problème est que l'expression référentielle est un substitut, et donc que ce dernier est supposé connu. Il l'est par inférence, comme on l'a dit, si l'on considère le titre... et la compétence particulière que le lecteur a de la structuration-composition des textes; et ici particulièrement des titres : n'est-il pas vrai que «[b]ien souvent, au simple aperçu du titre [...] on devine de quoi il va s'agir », comme Allais a pu l'écrire (Mobiliers militaires) ?

Dans ce cas, «Lui » suppose ou laisse supposer connue l'identité (nominale) du référent et joue donc un rôle d'anaphore ${ }^{25}$. Pour autant, il joue un second rôle référentiel-textuel, celui d'une forme de corrélat anaphorique ; autrement dit il est bi-endophorique : ana-cataphorique ou encore contrastif avec «Elle », qui est de même (je parle du référent) impliqué par Idylle ; c'est-à-dire qu'il est mobilisé, « activé » (COMBETTES, op. cit. : 57) topicalement dès le titre Idylle.

Quand «Lui » est (plutôt) non détaché, lié, on peut alors dire avec BlancheBenveniste (1997 : 110) que le pronom tonique «préfixe » gagne en familiarité ou en thématicité (vs en topicalité dans l'autre cas) : « il est prononcé sans saillance » (BLANCHE-BENVENISTE, op. cit. : 69).

\section{Pour conclure sur la perspective d'un autre traitement...}

Je ne vais pas répéter les conclusions que j'ai données dans les deux synthèses qui ont pris place dans ma partie précédente. En revanche, je reviendrai ici sur la

${ }^{25}$ Voir en note supra, la citation de CORNISH (2001: 5). 
perspective, l'ouverture dont je parlais à l'issue de la première partie; et, pour justifier le choix que j'ai fait d'un second traitement poétique au sens jakobsonien, je citerai l'auteur qui m'a inspiré, lui-même :

«Mais comment la poéticité se manifeste-t-elle ? En ceci, que le mot est ressenti comme mot et non comme simple substitut de l'objet nommé ni comme l'explosion d'émotion. En ceci, que les mots et leur syntaxe, leur signification, leur forme externe et interne ne sont pas des indices indifférents de la réalité, mais possèdent leur propre poids et leur propre valeur » (JAKOBSON, 1977 rééd. 2002 : 46).

Toutefois, et on s'en est déjà rendu compte, rien n'est «joué », car : «la fonction poétique organise et dirige l'œuvre poétique sans être nécessairement saillante et sans sauter aux yeux comme une affiche » (op. cit. : 47).

\section{BIBLIOGRAPHIE}

ACHARD-BAYLE Guy (éd. 2010), Linguistique textuelle : états de lieux, Verbum XXXII-2.

ACHARD-BAYle Guy \& ChABrolle-Cerretini Anne-Marie (éds. 2013), La Perspective fonctionnelle de la phrase (2) : du Cercle Linguistique de Prague à la Linguistique Textuelle, Verbum, XXXV 1-2 (voir infra en Webographie : Théories \& Concepts du Cercle Linguistique de Prague).

ADAM Jean-Michel (1997), Le style dans la langue, Lausanne, Delachaux \& Niestlé.

ARIEL Mira (1990), Accessing Noun Phrases Antecedents, Londres, Routledge.

BADIR Sémir (2014), Qu'est-ce qu'un thème? Une Approche sémiologique, comm. pers., publié ensuite (2015) dans Signata 5 (2014), http://www.presses.ulg.ac.be/jcms/c_14947/signata-volume-5-annales-dessemiotiques

BERRENDONNER Alain (1990), Pour une macro-syntaxe, Travaux de linguistique 21, p. 25-36.

BLANCHE-BENVENISTE Claire (1997), Approches de la langue parlée en français, Gap-Paris, Ophrys.

BLANCHE-BENVENISTE Claire et al. (1990), Le français parlé, Paris, Éditions du CNRS.

BLASCO-DulBECCO Mylène (1999), Les dislocations en français contemporain. Étude syntaxique, Paris, Honoré Champion.

Chambers Ross (1979), Parole et poésie, Poétique 37, p. 56-62.

COMBETTES Bernard (1983), Pour une grammaire textuelle. La progression thématique, Bruxelles Duculot, et Metz, Pratiques.

COMBETTES Bernard (1998), Les constructions détachées en français, Gap-Paris, Ophrys.

CORNISH Francis (2001), L'anaphore pronominale indirecte : une question de focus, in : De MULDER Walter, VeT Co \& VeTTERS Carl (éds), Anaphores pronominales et nominales: études pragma-sémantiques, Amsterdam, Rodopi, 1-25. 
DANEŠ František (1979 trad. fr. 2010), à propos de l'identification de l'information connue (contextuellement liée) dans le texte, in: ACHARDBAYLE Guy (éd.), Linguistique textuelle : états de lieux, Verbum $\mathrm{n}^{\circ}$ XXXII-2, p. 283-308.

DuBoIS Jean (1969), Grammaire générative et transformationnelle, Langue française 1, p. 49-57.

DUFRESNE Monique (1995), Étude diachronique de la cliticisation des pronoms sujets à partir du français médiéval, Revue québécoise de linguistique 24-1, p. 83-109.

Firbas Jan (1992), Functional Sentence Perspective in Written and Spoken Communication, Cambridge, Cambridge University Press.

HALLIDAY Michael A.K. (1985), Spoken \& Written Language, Oxford, Oxford University Press.

JAKOBSON Roman (1963), Les Fondations du langage. Essais de linguistique générale I, Paris, Les Éditions de Minuit, notamment le ch. 11 : Linguistique et poétique.

JAKOBSON Roman (1977 [Recueil de textes écrits entre 1919 et 1970] rééd. 2002), Huit questions de poétique, Paris, Éditions du Seuil, rééd. coll. Points-Essais (Voir particulièrement les chapitres: Qu'est-ce que la poésie ? p. 31-49, La Dominante p. 77-58, Poésie de la grammaire et grammaire de la poésie, p. 89126).

KRIPKE, Saul (1972 trad. fr. 1982), La Logique des noms propres, Paris, Les Éditions de Minuit.

KLEIBER Georges (1981), Problèmes de référence. Descriptions définies et noms propres, Recherches Linguistiques 6, Centre d'Analyse Syntaxique, Metz, et Paris, Klincksieck.

KLEIBER Georges (1994), Anaphores et pronoms, Louvain-la-Neuve, Duculot.

KLEIBER Georges (2001), Remarques sur la dénomination, Cahiers de Praxématique 36, p. 21-41.

KLINGLER Dominique (2011), Le marquage du topique en français (L2) et en japonais (L1) dans des productions bilingues de trois apprenantes avancées, Volume d'articles en vue de l'obtention de l'habilitation à diriger des recherches, Université de Provence (comm. pers).

LEVIN Samuel R. (1976), What Kind of a Speech Act a Poem Is?, in : VAN DIJK Teun A. (ed.), Pragmatics of Language \& Literature, Amsterdam, NorthHolland, p. 141-160.

MONTE Michèle (2012), Pour une autonomie relative des niveaux sémantique, énonciatif et iconique des textes poétiques, CMLF Congrès Mondial de Linguistique Française 2012,

http://www.shs-

conferences.org/articles/shsconf/abs/2012/01/shsconf_cmlf12_000044/shsconf _cmlf12_000044.html

PEŠEK Ondřej (2010), La linguistique textuelle tchèque au seuil du XXI siècle : la genèse d'une discipline et la tradition pragoise, Verbum XXXII-2, p. 263-282.

RADIMSKÝ Jan (éd. 2010), Centre-périphérie dans le système linguistique, Écho des Études Romanes EER VI 1-2, http://www.eer.cz/?s=2010_1-2 
Theissen Anne (2001), Petite incursion dans la jungle topicale, Cahiers de Praxématique37, http://praxematique.revues.org/200

VERONIQUE Georges Daniel (2013), La dislocation, l'emploi de c'est et il y a et l'organisation topique-focus en français parlé : des variétés d'apprenants aux usages natifs, Le Français parlé 29, 17 pages en ligne http://tipa.revues.org/1022

\section{Webographie (dont corpus)}

Alphonse ALLAIS, Idylle,http://www.bernard-joy.com/alphonse-allais-i/alphonseallais-idylle-recueil-a-1-oeil,a3427507.html

Alphonse ALLAIS, Idylle bourgeoise,http://www.bernard-joy.com/alphonse-allaisi/alphonse-allais-idylle-bourgeoise-recueil-a-l-oeil,a3413994.html

Alphonse ALLAIS, Idylle moderne, http://www.bernard-joy.com/alphonse-allaisi/alphonse-allais-idylle-moderne-recueil-pas-de-bile,a3592435.html

Cercle linguistique de Prague (Colloque De Nancy 2012 Theories \& CONCEPTS DU -)

http://www.atilf.fr/IMG/pdf/Colloque_CercleLinguistiquePrague_2012_Resumes. pdf

Robert DESNOS, Maudit soit l'homme, http://www.paperblog.fr/2495529/mauditrobert-desnos/ 